\title{
Myelitis Associated with Atopic Disorders in Japan: a Retrospective Clinical Study of the Past 20 Years
}

\author{
Jun-ichi Kira, Izumi Horiuchi, Jun Suzuki, Manabu Osoegawa, Shozo Tobimatsu*, Hiroyuki Murai, \\ Motozumi Minohara, Masutaka Furue** and Hirofumi OCHI
}

\begin{abstract}
Objective To clarify the clinical features of myelitis associated with atopic disorders in Japanese patients.

Subjects and Methods We retrospectively studied the clinical, immunological and electrophysiological features of 68 consecutive patients with myelitis of acute or subacute onset diagnosed at Kyushu University Hospital during the past 20 years.

Results While only 2 of $28(7 \%)$ patients with myelitis diagnosed between 1979 and 1993 had either atopic dermatitis (AD) or bronchial asthma (BA), 19 of 40 (48\%) patients with myelitis diagnosed between 1994 and 1998 did. Among the 40 patients with myelitis diagnosed between 1994 and 1998, 19 patients with either AD or BA as well as 21 patients without either disease showed a significantly higher level of serum total IgE, higher frequency of hyperIgEaemia and higher frequency of mite antigen-specific IgE than 82 healthy controls. Myelitis patients with AD presenting as persistent paresthesia/dysesthesia in all four limbs showed cervical cord lesions on MRI and abnormalities in upper limb motor evoked potentials but no abnormalities in the cerebrospinal fluid (CSF), while myelitis patients with BA showed preferential involvement of the lower motor neurons clinically and electromyographically. In addition, 12 patients with myelitis who had hyperIgEaemia and mite antigen-specific IgE but neither AD nor BA showed incomplete transverse myelitis with mild motor disability and few CSF abnormalities.

Conclusion The clinical features of myelitis associated with atopic disorders were in part distinguished by the type of preceding atopic disorder, and also were different from those of hyperIgEaemic myelitis with no preceding atopic disorders.
\end{abstract}

(Internal Medicine 40: 613-619, 2001)

Key words: Japanese, atopy, mite, atopic dermatitis, asthma, $\mathrm{IgE}$

\section{Introduction}

Atopy is characterized by enhanced IgE responses to such common environmental antigens as pollens, foods and house dust mites and predisposes humans to such atopic disorders as allergic rhinitis, bronchial asthma (BA) and atopic dermatitis (AD). The number of patients with atopic disorders has greatly increased in many industrialized countries including Japan (13 ). Although atopic disorders have never previously been shown to be associated with myelitis, we recently reported the occurrence of myelitis in adult patients with $\mathrm{AD}$ (4-6).

A poliomyelitis-like illness that presents as flaccid paralysis in one or more limbs after acute asthmatic attacks is known as asthmatic amyotrophy (Hopkins syndrome) (7). Although all previously reported cases were children younger than 13 years of age (7-21), we have recently seen adult patients with asthmatic amyotrophy (22). The observation that both atopic myelitis and asthmatic amyotrophy develop in the presence of atopic disorders prompted us to study more extensively the relationship between atopic disorders and the development of myelitis. We thus retrospectively studied patients diagnosed with acute or subacute myelitis of unknown etiology, clinically, immunologically, and electrophysiologically at Kyushu University Hospital over the past 20 years. The present study describes the clinical features of myelitis associated with atopic disorders in Japanese patients.

\section{Subjects and Methods}

\section{Subjects}

The subjects comprised 68 consecutive patients presenting with either acute or subacute onset of monophasic spinal cord syndrome who were subjected to a thorough neurological examination and routine laboratory tests at Kyushu University Hospital between 1979 and 1998. Patients who showed a stepwise progression over several months were also included because myelitic patients with AD have been reported to show such a clinical course (4-6). No specific cause was identified

From the Departments of Neurology and *Clinical Neurophysiology, Neurological Institute and **the Department of Dermatology, Graduate School of Medical Sciences, Kyushu University, Fukuoka

Received for publication July 12, 2000; Accepted for publication February 17, 2001

Reprint requests should be addressed to: Dr. Jun-ichi Kira, the Department of Neurology, Neurological Institute, Graduate School of Medical Sciences, Kyushu University, Maidashi 3-1-1, Fukuoka 812-8582 
in any of the patients, and all of them were diagnosed with acute or subacute myelitis of unknown etiology. The number of male patients was 38 and female patients, 30 . Their age at onset was 13 to 75 years old (mean age $\pm \mathrm{SD}=39 \pm 14$ years). For IgE assay, 43 patients (mean age $\pm \mathrm{SD}=50 \pm 7$ years, range 40-60 years) with neurodegenerative disorders such as Parkinson's disease, spinocerebellar degeneration or amyotrophic lateral sclerosis and 82 healthy subjects (mean age $\pm \mathrm{SD}=42 \pm 10$ years, range $22-58$ years) were included.

\section{Clinical studies}

All available clinical records were analyzed retrospectively. The disability of each patient was evaluated according to Kurtzke's expanded disability status scale (EDSS) (23). Corticosteroids (methylprednisolone pulse therapy $1 \mathrm{~g} \times 3$ days followed by $30-60 \mathrm{mg} /$ day oral prednisolone with gradual tapering) were considered effective when the patient showed improvement of 1 grade or more in EDSS scores.

Spinal cord magnetic resonance imaging (MRI) was done as described previously $(5,6)$. Briefly, T2-weighted (SE 2000$3000 / 80-102$ ) as well as T1-weighted (SE 400 or 500/16-20) images were obtained in the sagittal and axial planes using a high-field-strength (1.5 tesla) superconducting General Electric Signa MR imaging system. A contrast-enhanced study was also performed using the T1-weighted sequences.

\section{Electrophysiological studies}

Somatosensory evoked potentials (SEPs) and motor evoked potentials (MEPs) in upper and lower limbs were recorded as previously described $(24,25)$. In brief, the peak latencies of N9 (Erb's point), N13 (C7 cervical spine) and N20 (C3 or C4) were measured for the median SEPs. For the tibial SEPs, the peak latencies of $\mathrm{N} 20$ (T12 thoracic spine) and P37 (Cz') were measured. The upper limb (UL) MEPs were recorded from the abductor pollicis muscle by stimulating the hand motor area and cervical roots with a figure 8 -shaped magnetic coil. The lower limb (LL) MEPs were recorded from the abductor hallucis muscle by stimulating the leg motor area and lumbar roots. Evoked potentials were classified as abnormal if the latencies and central conduction time were delayed more than 3 SDs above normal or if any component was absent.

\section{Immunological studies}

The total and allergen-specific IgE levels in the serum taken during the acute or subacute stage were measured in 40 consecutive patients with myelitis who were studied between 1994 to 1998 by an enzyme-linked immunosorbent assay (ELISA) $(5,6)$. Two mite antigens, Dermatophagoides pteronyssinus and Dermatophagoides farinae, and cedar pollen were used as antigens for ELISA (AlaSTAT, Sankojunyaku, Tokyo). Patients with a serum IgE concentration higher than $250 \mathrm{U} / \mathrm{ml}$ were considered to have hyperIgEaemia as described previously ( 5 , 6). The cut-off value for allergen specific IgE was $0.34 \mathrm{IU} / \mathrm{ml}$.

\section{Statistics}

The Mann-Whitney U test was used for the statistical analysis of serum total IgE level, age of onset, EDSS scores, eosinophil percentage and CSF cell count and protein concentration. The chi-square test (or Fisher's exact test when the criteria of the chi-square test was not fulfilled) was used for the analysis of the frequency of hyperIgEaemia, allergen-specific IgE and other clinical and laboratory findings.

\section{Results}

\section{Frequency of associated atopic disorders by year of onset of myelitis}

Among the 68 patients with myelitis, 21 (31\%) had either AD or BA. As shown in Fig. 1, the total number of cases of myelitis as well as those complicated with $\mathrm{AD}$ or BA increased sharply after 1994. Thus, the frequency of coexistent AD or BA was significantly higher in the patients with myelitis seen between 1994 and 1998 (19 of 40,48\%) than in those between 1979 and $1993(2$ of $28,7 \%)(\mathrm{p}=0.0002)$.

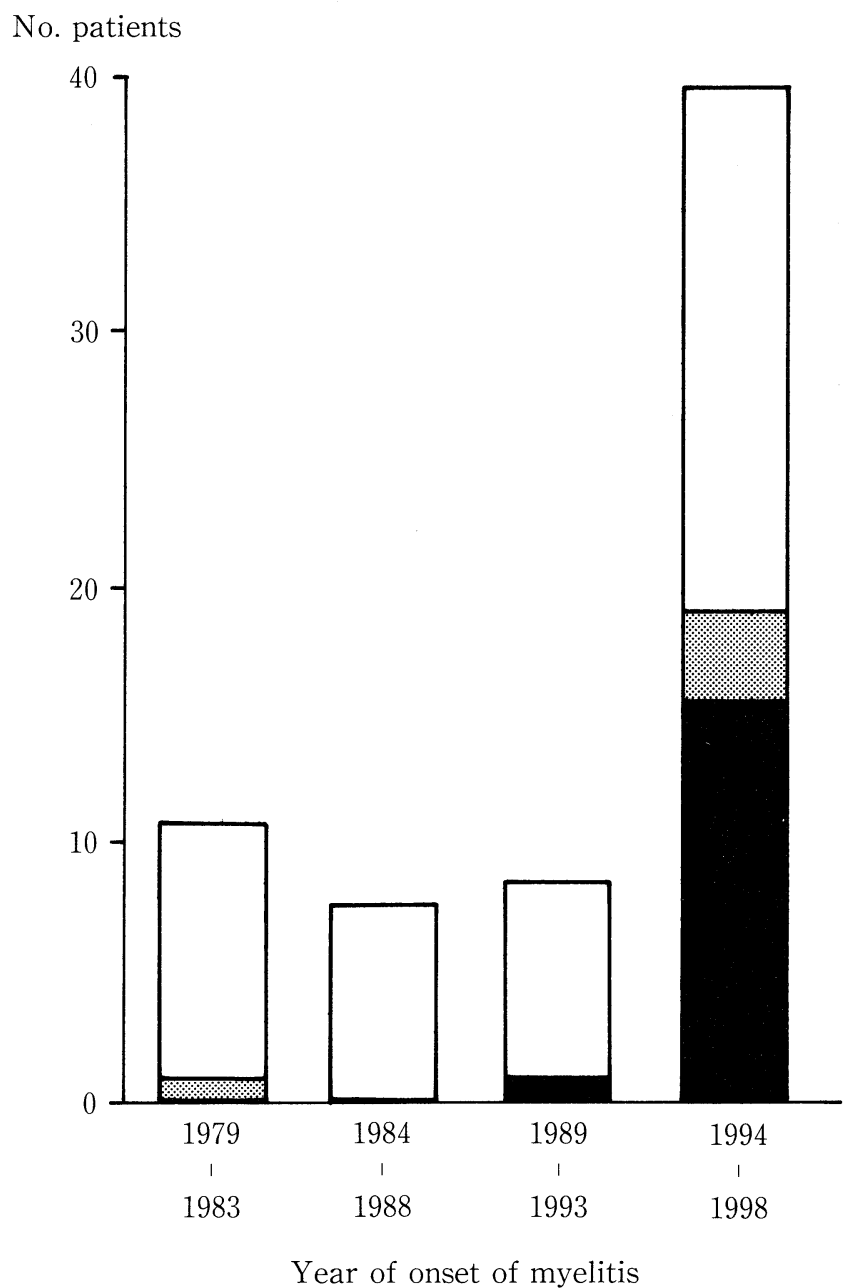

Figure 1. Frequency of associated atopic disorders by year of onset of myelitis. Black bar indicates myelitis with atopic dermatitis, hatched bar, myelitis with bronchial asthma, and white bar, myelitis without atopic disorders. 


\section{Myelitis with Atopic Disorders in Japan}

Comparison of clinical findings between myelitic patients with and without atopic disorders

Myelitis without such atopic disorders as AD and BA was more commonly seen in males, while myelitis with atopic disorders it was more frequent in females (Table 1). The age of onset was significantly younger in patients with atopic disorders than in those without atopic disorders $(\mathrm{p}=0.0011)$. The frequency of cervical cord involvement was significantly higher in patients with atopic disorders than in those without atopic disorders $(\mathrm{p}<0.0001)$, while the frequency of thoracic cord involvement was significantly higher in patients without atopic disorders than in those with atopic disorders ( $\mathrm{p}<0.0001)$. Myelitic patients with atopic disorders showed a significantly lower EDSS score than those without atopic disorders $(\mathrm{p}<0.0001)$. The frequency of CSF pleocytosis was significantly lower in the patients with atopic disorders than those without atopic dis- orders ( $\mathrm{p}=0.0390$ ). The CSF cell counts and total protein concentrations tended to be lower in the patients with atopic disorders than in those without atopic disorders $(\mathrm{p}=0.0781$ and $p=0.0649$, respectively). The frequency of eosinophilia in the peripheral blood in patients with atopic disorders was more than 4 times what it was in patients without atopic disorders $(\mathrm{p}<0.0001)$, and eosinophil percentages were significantly higher in the former compared to the latter $(\mathrm{p}=0.0005)$.

\section{Total and allergen-specific IgE}

The serum total IgE level as well as the frequency of hyperIgEaemia was significantly higher in all patients with myelitis $(\mathrm{p}<0.0001$ in either case) as well as in the myelitic patients with $(\mathrm{p}<0.0001$ in either case) and without atopic disorders $(\mathrm{p}<0.0001$ and $\mathrm{p}=0.0015$, respectively) in comparison to the healthy controls (Table 2). Moreover, the frequency of

Table 1. Comparison of Clinical Findings

\begin{tabular}{lcc}
\hline & \multicolumn{2}{c}{ Myelitis } \\
\hline & with AD or BA (n=21) & without AD or BA (n=47) \\
\hline Male/Female & $8 / 13(1: 1.63)$ & $30 / 17(1: 0.57)$ \\
Age of onset (mean \pm SD, yrs) & $32 \pm 13^{*}$ & $42 \pm 13$ \\
Clinically estimated main lesions ${ }^{\dagger}$ & & \\
$\quad$ Cervical cord & $20^{*}$ & 17 \\
$\quad$ Thoracic cord & $1^{*}$ & 29 \\
Lumbosacral cord & 2 & 4 \\
EDSS at peak & $2.0 \pm 1.0^{*}$ & $3.8 \pm 1.8$ \\
CSF & $2 \pm 2$ & $12 \pm 25$ \\
$\quad$ Cell counts $(/ \mu \mathrm{l})$ & $1 / 12(8 \%)^{*}$ & $20 / 44(45 \%)$ \\
Pleocytosis $(>5 / \mu \mathrm{l})$ & $37.6 \pm 20.8$ & $45.9 \pm 22.7$ \\
Total protein amounts $(\mathrm{mg} / \mathrm{dl})$ & $3 / 12(25 \%)$ & $25 / 44(57 \%)$ \\
Increased protein $(>40 \mathrm{mg} / \mathrm{dl})$ & $11.1 \pm 9.5 *$ & $3.9 \pm 3.3$ \\
Eosinophil $(\%)$ & $14 / 19(74 \%)^{*}$ & $8 / 44(18 \%)$ \\
Eosinophilia $(>5 \%)$ & & \\
\hline
\end{tabular}

$\dagger 3$ patients without atopic disorders and 2 patients with atopic disorders showed the main lesions at two distinct or consecutive levels of the spinal cord. *The difference is statistically significant between the two groups $(\mathrm{p}<0.05)$. EDSS: Kurtzke's expanded disability status scale, AD: atopic dermatitis, BA: bronchial asthma.

Table 2. Frequency of HyperIgEaemia and Allergen Specific IgE

\begin{tabular}{|c|c|c|c|c|c|}
\hline & \multirow{2}{*}{$\begin{array}{c}\text { IgE } \\
\text { (median, U/ml) }\end{array}$} & \multirow{2}{*}{ HyperIgEaemia } & \multicolumn{3}{|c|}{ Allergen specific IgE } \\
\hline & & & D. pteronyssinus & D. farinae & Cedar pollen \\
\hline \multicolumn{6}{|l|}{ Myelitis } \\
\hline total $(n=40)$ & $364 *$ & $29 / 40(73 \%)^{*}$ & $32 / 40(80 \%)^{*}$ & $29 / 40(73 \%)^{*}$ & $22 / 40(55 \%)$ \\
\hline with $\mathrm{AD}$ or $\mathrm{BA}(\mathrm{n}=19)$ & $1,150 *$ & $17 / 19(89 \%)^{* 8}$ & $17 / 19(89 \%)^{*}$ & $17 / 19(89 \%)^{* 8}$ & $15 / 19(79 \%) * \S$ \\
\hline without $\mathrm{AD}$ or $\mathrm{BA}(\mathrm{n}=21)$ & $238^{*}$ & $12 / 21(57 \%)^{*}$ & $15 / 21(71 \%)^{*}$ & $12 / 21(57 \%)^{*}$ & $7 / 21(33 \%)$ \\
\hline Neurodegeneration $(n=43)$ & 47 & $10 / 43(23 \%)$ & $17 / 43(40 \%)$ & $13 / 43(30 \%)$ & $22 / 43(51 \%)$ \\
\hline Healthy controls $(n=82)$ & 79 & $17 / 82(21 \%)$ & $26 / 82(32 \%)$ & $25 / 82(30 \%)$ & $41 / 82(50 \%)$ \\
\hline
\end{tabular}

${ }^{*} \mathrm{p}<0.05$, in comparison to healthy controls. ${ }^{\S} \mathrm{p}<0.05$, in comparison between myelitis with AD or BA and that without AD or BA. AD: atopic dermatitis, BA: bronchial asthma, D.: Dermatophagoides. 
Dermatophagoides pteronyssinus- and Dermatophagoides farinae-specific IgE was significantly higher in all patients with myelitis ( $p<0.0001$ for either antigen), myelitic patients with atopic disorders ( $<<0.0001$ for either antigen) and those without atopic disorders ( $\mathrm{p}=0.0010$ and 0.0257 , respectively) than in the healthy controls. The frequency of cedar pollen-specific IgE was also significantly higher only in the myelitic patients with atopic disorders than in the healthy controls $(\mathrm{p}=0.0181)$. Among the myelitic patients, those with atopic disorders showed a significantly higher level of serum $\operatorname{IgE}(\mathrm{p}=0.0008)$, higher frequencies of hyperIgEaemia $(\mathrm{p}=0.0181)$ and Dermatophagoides farinae- and cedar pollen-specific $\operatorname{IgE}(\mathrm{p}=0.0181$ and $\mathrm{p}=0.0031$, respectively) than those without atopic disorders. One patient with $\mathrm{AD}$ and another patient with $\mathrm{BA}$ had no IgE antibodies to mite antigens, but the $\mathrm{AD}$ patient had $\mathrm{IgE}$ specific to egg white, which was the responsible allergen for the AD in her case, and the BA patient had cedar pollen specific IgE. As a result, among 40 patients with myelitis seen between 1994 and 1998, 16 (40\%) had AD, 3 (7.5\%) had BA, $12(30 \%)$ had hyperIgEaemia in the absence of AD or BA, and only $9(22.5 \%)$ had neither hyperIgEaemia nor such atopic disorders. Patients with neurodegenerative disorders showed no significant difference in the frequency of hyperIgEaemia or any allergen specific IgE, in comparison to the healthy controls.

\section{Comparison of clinical and laboratory findings}

Forty-five patients whose IgE status was examined during the acute stage were divided into the following 4 groups and their clinical and laboratory findings were compared (Table 3 ); myelitis with $\mathrm{AD}$ (group 1), myelitis with BA (group 2), myelitis with hyperIgEaemia but without AD or BA (group 3), and myelitis without hyperIgEaemia, AD or BA (group 4).

Groups 3 and 4 were preponderantly male while no such trend was found in groups 1 and 2. In group 1, 7 of 17 patients developed neurologic symptoms immediately after an exacerbation of $\mathrm{AD}$, and all 3 patients in group 2 developed myelitis following acute asthma attacks. In group 1, paresthesia/dysesthesia in the distal parts of all four limbs was the most common and predominant symptom throughout the entire clinical course. Mild to moderate hyperreflexia in all four limbs was frequently found in this group, yet pathological reflex and apparent muscle weakness were rare. On the contrary, 3 patients in group 2 commonly presented with moderate to severe motor weakness and later developed apparent muscle atrophy. The lesions were clinically estimated to be in the cervical cord in all patients of group 1, whereas no such trend of localization was found in groups 2, 3 or 4. EDSS at peak was 4 or less in groups 1,2 and 3 , whereas 5 of 13 patients in group 4 showed EDSS scores higher than 4.5.

CSF pleocytosis and protein increase were frequently seen in group 4, whereas they were absent in group 1 and infrequent in groups 2 and 3 . An increased IgG index and oligoclonal bands were rare in all 4 groups. Spinal cord MRI confirmed the predominant involvement of the cervical cord in the group 1, but not in any other groups.
In group 4, both MEPs and SEPs were more frequently abnormal in the lower limbs than in the upper limbs, suggesting severe involvement of the thoracic cord. In group 3, the lower limbs also tended to show abnormalities in MEPs and SEPs more frequently than the upper limbs. In contrast, the central motor conduction time (CMCT) of UL-MEPs was prolonged with normal F-wave latency in 5 of 8 patients (or 6 limbs) in group 1 . However, only one patient showed bilateral prolonged CMCT in LL-MEPs. In addition, 2 of 8 patients showed unilateral prolonged N20 latency with absent N13 in median nerve SEPs, while only one patient showed the unilateral absence of P37 with normal N20 in tibial nerve SEPs. Thus, in group 1, cervical cord lesions were documented in 10 of 17 patients (59\%) by MRI and/or evoked potentials ( 6 by MRI and MEPs, 2 by MRI alone and 2 by MEPs alone). Needle EMG disclosed on-going denervation potentials in the affected limbs in all patients examined in group 2 , whereas such findings were absent in group 1 , infrequent in group 4 , and only occasionally found in group 3.

Paresthesia/dysesthesia was persistent in most patients in group 1, and motor weakness persisted with no improvement in group 2. Corticosteroids were less effective and the deficits persisted more frequently in group 3 , whereas clinical improvement was seen more frequently and corticosteroids were considered to be effective in group 4 .

\section{Discussion}

The main new findings in the present study are: 1) myelitis without AD or BA showed a significantly higher level of serum total IgE, a higher frequency of hyperIgEaemia and a higher frequency of mite antigen-specific IgE than the healthy controls, 2) hyperIgEaemic myelitis without $\mathrm{AD}$ or BA showed some features common to hyperIgEaemic myelitis with $\mathrm{AD}$ or BA, i.e., persistent spinal cord symptoms, low EDSS scores, low frequency of CSF abnormalities and lower motor neuron involvement as shown by needle EMG, 3) myelitis with $\mathrm{AD}$ frequently showed abnormalities in upper limb MEPs, 4) myelitis with atopic disorders has recently increased.

As reported previously $(4-6,22)$, myelitis with $\mathrm{AD}$ involved the posterior column of the cervical cord, as shown clinically and by MRI, whereas myelitis with BA showed involvement of anterior horn cells. Because all of the present patients with BA had hyperIgEaemia and allergen-specific IgE, the preceding asthma attacks were most likely atopic. It is noteworthy that myelitis frequently developed after an exacerbation of $\mathrm{AD}$ or BA in the present series. Both AD and BA are induced immunologically in Th2 cell overactive conditions (26). Thus, myelitis in the patients with these atopic disorders appears to have commonly developed in the state of Th2 cell hyperactivity. This clearly distinguishes it from MS, which has been reported to be attenuated in Th2 cell overactive conditions such as pregnancy (27) and atopic disorders (28). This observation is explained by the fact that $\mathrm{Th} 1$ and $\mathrm{Th} 2$ cells cross-regulate each other and by the current hypothesis that MS is caused by autoimmune Th1 cells reacting against myelin (29). In the case 
Myelitis with Atopic Disorders in Japan

Table 3. Comparison of Clinical and Laboratory Findings

\begin{tabular}{|c|c|c|c|c|}
\hline & \multicolumn{3}{|c|}{ Myelitis with atopy } & \multirow{2}{*}{$\begin{array}{c}\text { Myelitis without atopy } \\
\text { without AD, BA and } \\
\text { hyperIgEaemia }(n=13)\end{array}$} \\
\hline & $\begin{array}{l}\text { with } \mathrm{AD} \\
(\mathrm{n}=17)\end{array}$ & $\begin{array}{l}\text { with BA } \\
\qquad(n=3)\end{array}$ & $\begin{array}{l}\text { with hyperIgEaemia } \\
\text { alone }(n=12)\end{array}$ & \\
\hline Male/Female & $6 / 11$ & $1 / 2$ & $9 / 3$ & $8 / 5$ \\
\hline $\begin{array}{l}\text { Age of onset (mean } \pm \mathrm{SD}, \mathrm{yrs}) \\
\quad \text { (range) }\end{array}$ & $\begin{array}{c}30 \pm 8 \\
(20-46)\end{array}$ & $\begin{array}{c}37 \pm 26 \\
(15-73)\end{array}$ & $\begin{array}{c}36 \pm 10 \\
(15-46)\end{array}$ & $\begin{array}{c}43 \pm 17 \\
(13-75)\end{array}$ \\
\hline \multicolumn{5}{|l|}{ Clinical course } \\
\hline monophasic & 7 & 2 & 6 & 9 \\
\hline step-wise progression/fluctuation & 7 & 1 & 6 & 4 \\
\hline \multicolumn{5}{|l|}{ Symptoms and signs } \\
\hline paresthesia/dysesthesia & 16 & 1 & 10 & 13 \\
\hline increased tendon reflex & 13 & 2 & 8 & 12 \\
\hline sensory signs & 7 & 1 & 8 & 10 \\
\hline sphincter disturbance & 1 & 0 & 7 & 7 \\
\hline Lhermitte sign & 4 & 1 & 2 & 3 \\
\hline motor weakness & 2 & 3 & 6 & 7 \\
\hline muscle atrophy & 0 & 3 & 0 & 1 \\
\hline pathological reflexes & 1 & 0 & 5 & 7 \\
\hline \multicolumn{5}{|l|}{ Clinically estimated lesions } \\
\hline cervical cord & 17 & 2 & 6 & 7 \\
\hline thoracic cord & 0 & 1 & 6 & 7 \\
\hline lumbosacral cord & 0 & 2 & 0 & 1 \\
\hline EDSS at peak & $1.7 \pm 0.9$ & $3.2 \pm 0.2$ & $3.0 \pm 0.8$ & $4.5 \pm 2.6$ \\
\hline (range) & $(1.0-3.5)$ & $(3.0-3.5)$ & $(1.0-4.0)$ & $(1.0-9.0)$ \\
\hline Eosinophilia (>5\%) & $11 / 15$ & $2 / 3$ & $4 / 11$ & $1 / 12$ \\
\hline \multicolumn{5}{|l|}{ CSF } \\
\hline pleocytosis $(>5 / \mu 1)$ & $0 / 8$ & $1 / 3$ & $2 / 10$ & $8 / 13$ \\
\hline increased protein $(>40 \mathrm{mg} / \mathrm{dl})$ & $0 / 8$ & $2 / 3$ & $4 / 10$ & $9 / 13$ \\
\hline oligoclonal bands & $0 / 8$ & $0 / 2$ & $0 / 9$ & $1 / 10$ \\
\hline increased $\mathrm{IgG}$ index & $0 / 8$ & $1 / 2$ & $1 / 9$ & $1 / 10$ \\
\hline \multicolumn{5}{|l|}{ Spinal cord MRI } \\
\hline T2-high lesions & $8 / 17$ & $1 / 3$ & $7 / 12$ & $9 / 13$ \\
\hline cervical cord & 8 & 1 & 4 & 6 \\
\hline thoracic cord & 1 & 0 & 4 & 6 \\
\hline lumbosacral cord & 0 & 0 & 0 & 0 \\
\hline Gadolinium enhancement & 3 & 1 & 6 & 5 \\
\hline \multicolumn{5}{|l|}{ Motor evoked potentials } \\
\hline upper limbs & $5 / 8$ & $1 / 2$ & $1 / 9$ & $2 / 8$ \\
\hline lower limbs & $1 / 8$ & $1 / 2$ & $3 / 10$ & $4 / 9$ \\
\hline \multicolumn{5}{|l|}{ Somatosensory evoked potentials } \\
\hline upper limbs & $2 / 8$ & $1 / 1$ & $2 / 7$ & $2 / 8$ \\
\hline lower limbs & $1 / 8$ & $0 / 2$ & $3 / 8$ & $8 / 12$ \\
\hline \multicolumn{5}{|l|}{ Needle EMG } \\
\hline on-going denervation potentials & $0 / 4$ & $3 / 3$ & $2 / 5$ & $1 / 6$ \\
\hline giant motor unit potentials & $0 / 4$ & $2 / 3$ & $3 / 5$ & $1 / 6$ \\
\hline \multicolumn{5}{|l|}{ Outcome } \\
\hline unimproved & $9 / 17$ & $3 / 3$ & $4 / 12$ & $2 / 13$ \\
\hline improved with corticosteroids & $2 / 4$ & $0 / 2$ & $5 / 9$ & $8 / 10$ \\
\hline
\end{tabular}

EDSS: Kurtzke's expanded disability status scale, AD: atopic dermatitis, BA: bronchial asthma.

of myelitis with atopic disorders, the occurrence of CNS inflammation in the Th2 hyperactive state suggests a distinct mechanism to be operative.

Another important finding is that myelitis without AD or
BA was also associated with hyperIgEaemia and mite antigenspecific IgE. Therefore, some hyperIgEaemic myelitis patients without the preceeding $\mathrm{AD}$ or BA may also have a mechanism of spinal cord inflammation similar to those with $\mathrm{AD}$ or $\mathrm{BA}$. 
Recently myelitis with a preceding history of $\mathrm{AD}$ has been reported to show an inflammatory lesion with marked eosinophil infiltration in the biopsied spinal cord (30). Although it is still uncertain as to whether or not all three myelitic conditions with hyperIgEaemia, i.e., myelitis with $\mathrm{AD}$, myelitis with $\mathrm{BA}$ and hyperIgEaemic myelitis without $\mathrm{AD}$ or $\mathrm{BA}$, have a similar underlying pathomechanism, it is interesting to note that all three conditions frequently show atopy to environmental allergens as well as a tendency toward eosinophilia. Eosinophils harboring high-affinity IgE receptors are known to play a key role in persistent inflammation (late-phase reaction of type 1 allergy) in BA as well as in AD (26). Therefore, in such myelitic conditions with atopic disorders, it is possible that eosinophils infiltrating from the peripheral blood into the spinal cord may contribute to the development of inflammation.

The present study is the first to describe the electrophysiological findings in myelitis with $\mathrm{AD}$. In this condition, MEPs were more frequently abnormal than SEPs; however, there were more patients with sensory symptoms than with motor disturbance. Prolonged CMCT in UL-MEPs with normal F-wave latency may indicate corticospinal tract involvement in the cervical cord while an absent N13 in median nerve SEPs suggests dysfunction of the posterior column. A high frequency of MEP abnormalities is likely to correspond to hyperreflexia in the upper limbs while a low frequency of SEP abnormalities may reflect a mild degree of posterior column dysfunction (predominantly paresthesia/dysesthesia but rarely sensory ataxia). These neurophysiological findings may correspond to the high signal intensity lesions in the cervical cord on T2-weighted MRI. The observation that the frequency of abnormal UL-MEPs was higher than that of LL-MEPs is in direct contrast to the results in MS (31). The present results suggest that UL-MEPs together with cervical MRI are useful to localize the responsible lesions in myelitis with $\mathrm{AD}$.

The results of the present study indicate the recent emergence of myelitis with atopic disorders in Japan. The occurrence of myelitis with atopic disorders has been reported in other districts of Japan $(32,33)$ since our first report (4). However, the retrospective nature of the present study as well as the recent awareness of myelitis with atopic disorders may well have increased the frequency of diagnosis of myelitis with atopic disorders over the past 5 years, as reflected in the present study. Therefore, our findings concerning the recent increase of myelitis with atopic disorders should be interpreted cautiously. In order to further clarify the relationship between atopy and myelitis, a large-scale epidemiological survey is necessary. Since the number of adult patients with atopic disorders is greatly increasing in many industrialized countries now, it is important in clinical practice to be aware of the possibility of encountering myelitis associated with atopic disorders.

Acknowledgements: This work was supported in part by Grants 10470154, 10557062 and 10877099 from the Ministry of Education, Science, Sports and Culture of Japan, and grants from the Neuroimmunological Disease Research Committee, the Ministry of Health and Welfare of Japan. We thank Ms. Akane Nakamura for typing assistance.

\section{References}

1) Rothe MJ, Grant-Kels JM. Atopic dermatitis: an update. J Am Acad Dermatol 35: 1-13, 1996.

2) Hopkin JM. Mechanisms of enhanced prevalence of asthma and atopy in developed countries. Curr Opin Immunol 9: 788-792, 1997.

3) Sugiura H, Umemoto N, Deguchi H, et al. Prevalence of childhood and adolescent atopic dermatitis in a Japanese population: comparison with the disease frequency examined 20 years ago. Acta Derm Venereol 78: 293-294, 1998.

4) Kira J, Yamasaki K, Kawano Y, Kobayashi T. Acute myelitis associated with hyperIgEemia and atopic dermatitis. J Neurol Sci 148: 199-203, 1997.

5) Kira J, Kawano Y, Yamasaki K, Tobimatsu S. Acute myelitis with hyperIgEaemia and mite antigen specific IgE: atopic myelitis. J Neurol Neurosurg Psychiatry 64: 676-679, 1998.

6) Kira J, Kawano Y, Horiuchi I, et al. Clinical, immunological and MRI features of myelitis with atopic dermatitis (atopic myelitis). J Neurol Sci 162: 56-61, 1999.

7) Hopkins IJ. A new syndrome: poliomyelitis-like illness associated with acute asthma in childhood. Aust Paediatr J 10: 273-276, 1974.

8) Hopkins IJ, Shield LK. Poliomyelitis-like illness associated with asthma in childhood. Lancet i: 760, 1974.

9) Danta G. Electrophysiological study of amyotrophy associated with acute asthma (asthmatic amyotrophy). J Neurol Neurosurg Psychiatry 38: 1016$1021,1975$.

10) Ilett SJ, Pugh RJ, Smithells RW. Poliomyelitis-like illness after acute asthma. Arch Dis Child 52: 738-740, 1977.

11) Warren P, Fischbein C, Mascoli N, Rudolph J, Hodder DH. Poliomyelitis-like syndrome caused by mycoplasma pneumoniae. J Pediatr 93: 451452,1978 .

12) Shapiro GG, Chapman JT, Pierson WE, Bierman CW. Poliomyelitis-like illness after acute asthma. J Pediatr 94: 767-768, 1979.

13) Blomquist HKS, Björkstén B. Poliomyelitis-like illness associated with asthma. Arch Dis Child 55: 61-63, 1980.

14) Manson JI, Thong YH. Immunological abnormalities in the syndrome of poliomyelitis-like illness associated with acute bronchial asthma (Hopkins's syndrome). Arch Dis Child 55: 26-32, 1980.

15) Wheeler SD, Ochoa J. Poliomyelitis-like syndrome associated with asthma: a case report and review of the literature. Arch Neurol 37: 52-53, 1980.

16) Shahar EM, Hwang PA, Niesen CE, Murphy EG. Poliomyelitis-like paralysis during recovery from acute bronchial asthma: possible etiology and risk factors. Pediatrics 88: 276-279, 1991.

17) Kyllerman MG, Herner $S$, Bergström TB, Ekholm SE. PCR diagnosis of primary herpes virus type $I$ in poliomyelitis-like paralysis and respiratory tract disease. Pediatr Neurol 9: 227-229, 1993.

18) Liedholm LJA, Eeg-Olofsson O, Ekenberg BEK, Nicolaysen RB, Torbergsen T. Acute postasthmatic amyotrophy (Hopkins' syndrome). Muscle Nerve 17: 769-772, 1994.

19) Arita J, Nakae Y, Matsushima H, Maekawa $K$. Hopkins syndrome: $T_{2^{-}}$ weighted high intensity of anterior horn on spinal MR imaging. Pediatr Neurol 13: 263-265, 1995.

20) Mizuno Y, Komori S, Shigetomo R, Kurihara E, Tamagawa K, Komiya K. Poliomyelitis-like illness after acute asthma (Hopkins syndrome): a histological study of biopsied muscle in a case. Brain Dev 17: 126-129, 1995.

21) Acharya AB, Lakhani PK. Hopkins syndrome associated with mycoplasma infection. Pediatr Neurol 16: 54-55, 1997.

22) Horiuchi I, Yamasaki K, Osoegawa M, et al. Acute myelitis following asthma attacks with onset after puberty. J Neurol Neurosurg Psychiatry 68: 665-668, 2000.

23) Kurtzke JF. Rating neurologic impairment in multiple sclerosis: an expanded disability status scale (EDSS). Neurology 33: 1444-1452, 1983.

24) Kira J, Tobimatsu S, Goto I, Hasuo K. Primary progressive versus relaps- 


\section{Myelitis with Atopic Disorders in Japan}

ing remitting multiple sclerosis in Japanese patients: a combined clinical, magnetic resonance imaging and multimodality evoked potential study. J Neurol Sci 117: 179-185, 1993.

25) Tobimatsu S, Sun S-J, Fukui R, Kato M. Effects of sex, height and age on motor evoked potentials with magnetic stimulation. J Neurol 245: 256261, 1998.

26) Rudikoff D, Lebwohl M. Atopic dermatitis. Lancet i: 1715-1721, 1998.

27) Birk K, Rudick R. Pregnancy and multiple sclerosis. Arch Neurol 43: 719-726, 1986.

28) Kira J, Kawano Y, Yamasaki K. Multiple sclerosis with mite antigenspecific IgE. J Neurol Sci 157: 138-142, 1998.

29) Miller SD, Karpus WJ. The immunopathogenesis and regulation of T- cell-mediated demyelinating diseases. Immunol Today 15: 356-361, 1994.

30) Kikuchi H, Osoegawa M, Ochi H, et al. Spinal cord lesions of myelitis with hyperIgEaemia and mite antigen specific IgE (atopic myelitis) manifest eosinophilic inflammation. J Neurol Sci 183: 73-78, 2001.

31) Eisen AA, Shtybel W. AAEM Minimonograph \#35: Clinical experience with transcranial magnetic stimulation. Muscle Nerve 13: 995-1011, 1990.

32) Hinuma $Y$, Sato $S$, Kato $H$, Itoyama $Y$. A case of thoracic myelitis associated with hyperIgEaemia and eosinophilia. Neuroimmunology 6: 81-85, 1998 (in Japanese, Abstract in English).

33) Horinouchi $H$, Inobe J, Mori $T$, Kumamoto $T$, Tsuda $T$. A case of atopic myelitis. Rinshou Shinkeigaku (Clin Neurol) 40: 218-221, 2000 (in Japanese, Abstract in English). 